\title{
Mathematical Features for Recognizing Preference in Sub-Saharan African Traditional Rhythm Timelines
}

\author{
Godfried Toussaint* \\ School of Computer Science \\ McGill University \\ Montréal, Québec, Canada
}

\begin{abstract}
The heart of an African rhythm is the timeline, a beat that cyclically repeats thoughout a piece, and is often performed with an iron bell that all performers can hear. Such rhythms can be represented as sequences of points on a circular lattice, where the position of the points indicates the time in the cycle at which the instrument is struck. Whereas in theory there are thousands of possible choices for such timeline patterns, in practice only a few of these are ever used. This brings up the question of how these few patterns were selected over all the others, and of those selected, why some are preferred (have more widespread use) than others. Simha Arom discovered that the rhythms used in the traditional music of the Aka Pygmies of Central Africa possess what he calls the rhythmic oddity property. A rhythm has the rhythmic oddity property if it does not contain two onsets that partition the cycle into two half-cycles. Here a broader spectrum of rhythms from West, Central and South Africa are analysed. A mathematical property of rhythms is proposed, dubbed "Off-Beatness", that is based on group theory, and it is argued that it is superior to the rhythmic oddity property as a measure of preference among Sub-Saharan African rhythm timelines. The "Off-Beatness" measure may also serve as a mathematical definition of syncopation, a feature for music recognition in general, and it is argued that it is superior to the mathematical syncopation measure proposed by Michael Keith.
\end{abstract}

\section{Introduction}

It is useful for the mathematical analysis of cyclic rhythms to represent them as ordered sets of points on a circle. The points represent the onsets of notes in time. For example, the rhythm consisting of five onsets with corresponding time intervals of ( $\left.\begin{array}{lllll}3 & 2 & 3 & 2 & 2\end{array}\right)$ units is illustrated in Figure 1 (a), where the onsets, starting at position "zero", are joined together with straight line segments to form a convex polygon. This rhythm is used in the traditional music of the Aka Pygmies of Central Africa [1], [2], [3]. The rhythm contains two intervals

^ Research supported by NSERC. e-mail: godfried@cs.mcgill.ca 


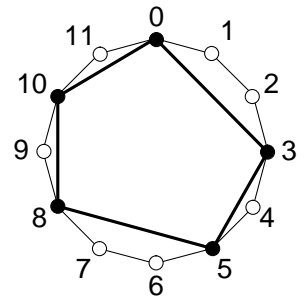

(a)

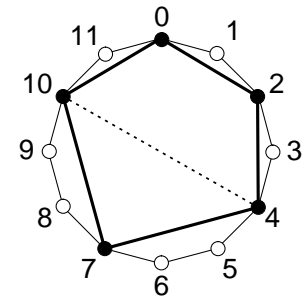

(b)

Fig. 1. (a) A rhythm used by the Aka Pygmies of Central Africa, (b) The Seguiriya rhythm used in the Flamenco music of Spain.

of length three and three intervals of length two. The interval lengths consisting of one, two, and three units are strongly favoured in the rhythm timelines of much of Sub-Saharan traditional music [7], [8], [9]. Given only this constraint, there are usually different ways of arranging the intervals while maintaining their cardinality fixed (cyclic permutations). For example, in the preceeding rhythm of Figure 1 (a) the two intervals of length three may be placed side by side as in Figure 1 (b). The resulting rhythm, with time intervals (interval vector) given by (2 22332 ), is the Seguiriya metric pattern used in the Flamenco music of Andalucia in Southern Spain [4].

The question then arizes as to how the $A k a$ Pygmies evolved to prefer the first of these two rhythms over the second. Simha Arom [1] discovered that the $A k a$ Pygmies use rhythms that have the rhythmic-oddity property [2], [3]. A rhythm consisting of an even number of time units, has the rhythmic-oddity property if no two onsets partition the cycle (entire time span) into two sub-intervals of equal length. Such a partition will be called an equal bi-partition. Note that the rhythm of the Aka Pygmies in Figure 1 (a) has the rhythmic-oddity property, whereas the Seguiriya rhythm of Figure 1 (b) does not.

A note is in order to clarify what we mean when we write that one rhythm is "preferred" over another. This preference has not been established scientifically in a laboratory by experiments conducted by music perception psychologists. Rather, it is inferred from the existence and ubiquity of the rhythms observed in the field by ethnomusicologists [1]. It is assumed for example that rhythms that are played, are preferred over those that are not.

Before proceeding it is useful to define some terminology. A necklace token is a circular chain of $n$ elements of $m$ different colors. Two $n$-element necklace tokens are considered to be one and the same necklace if one token can be rotated so that there is a one-to-one correspondence between the colors of their elements. However, a necklace token may not be turned over to find such a correspondence [6]. A Lyndon word is a non-periodic necklace [6]. 


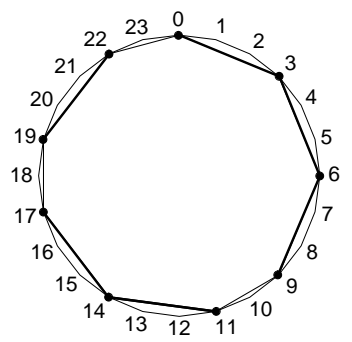

Fig. 2. The Mokongo rhythm used by the Aka Pygmies.

The two rhythms depicted in Figure 1 with interval vectors (3 22322 ) and (2 2333 2) can be viewed as necklaces, where the intervals of the rhythms correspond to the elements of the necklaces and the two different lengths (2 and 3 units) of the intervals correspond to the two colors of the elements. In other words, both rhythms are distinct 5-element necklaces of two different colors with the additional constraint that the color of the 3-unit intervals is used exactly twice. This additional constraint is called fixed density. It is an interesting computational problem to generate efficiently all necklaces and Lyndon words under these constraints [10]. It follows from Pólya's Theorem [6], and the reader may easily verify, that the two rhythms of Figure 1 are the only necklaces possible composed of five elements of two colors with exactly two elements of one color. Both rhythms are also Lyndon words (non-periodic).

The Aka Pygmies also play two other rhythm timelines that use intervals of length two and three units in a time span of 24 units [1], [2], [3]. The first is the pattern (32222322222) containing two intervals of length three and nine intervals of length two. There are five distinct necklaces of eleven elements of two colors with exactly two elements of one color. Using interval vector notation the five necklaces are: (3 3222222222$)$, (3 23222222222 ), (3 223222222 2), (3 222322222 2), (3 222232222 2). Only the last one of these, selected by the $A k a$ Pygmies, has the rhythmic oddity property, and none of the other four patterns are used by the $A k a$ Pygmies.

The second 24-unit pattern used by the Aka Pygmies is ( $\begin{aligned} & 3 \\ & 3\end{aligned} 3233232$ ) which contains six intervals of length three and three intervals of length two. This rhythm depicted in Figure 2 is called the Mokongo, and also has the rhythmic oddity property.

One might hypothesise that the rhythmic-oddity property is a good measure of preference used as a selection criterion (perhaps unconciously) for the adoption of rhythms by the peoples of Central Africa in general, and the Aka Pygmies in particular. One might even hope that this property could predict rhythm preference in other parts of Africa. However, for these purposes the rhythmicoddity property has some limitations. 
Consider for example the ten fundamental West (and South) African bell timelines composed of seven onsets in a time span of twelve units, with five intervals of length two and two intervals of length one (see [12] for more details).

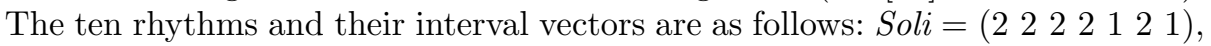

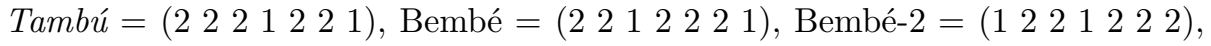

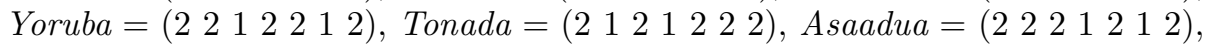

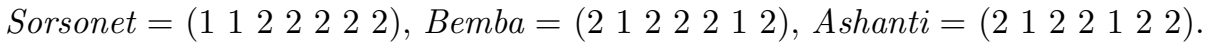
All ten rhythms are obtained by suitable rotations of one of three necklaces as pictured in Figure 3. None of these ten rhythms, nor any of the other eleven that are not used but belong to the same three generating necklaces, has the rhythmic-oddity property. Furthermore, among the group of ten that are used, some are more widespread than others, but the rhythmic oddity property does not offer an explanation for this preference.

Turning to the Mokongo rhythm of the Aka Pygmies, there are ten distinct necklaces of nine elements of two colors with exactly six elements of one color. Using interval vector notation the ten necklaces are:

(3 33333322 2), (3 33333232 2), (3 333332232 ), (3 33332332 2), (3 33332323 ), (3 33332233 2), (3 33323332 2), (3 33323323 2),

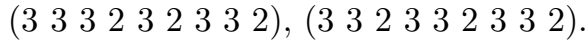

The last of these is periodic and therefore not of interest in this context. Of the nine distinct Lyndon words remaining, the last three have the rhythmic oddity property. Therefore the rhythmic-oddity property fails to explain how the Mokongo pattern is favored over the other two.

Here a broader spectrum of rhythm timelines from West, Central, and South Africa are considered. A mathematical property of rhythms is proposed, dubbed "off-beatness", based on group generators, and it is argued that it is superior to the rhythmic-oddity property as a measure of preference in Sub-Saharan African rhythm. First we take a small detour to describe a generalization of the rhythmic oddity property that widens its applicability.

\section{A Generalization of the Rhythmic Oddity Property}

Simha Arom [1] defines the rhythmic oddity property in a strictly binary mode, i.e., a rhythm either has or does not have the rhythmic oddity property. This concept may be generalized by defining a multi-valued variable that measures the amount of rhythmic oddity a rhythm possesses. This variable (rhythmic oddity) is defined as the number of equal bi-partitions that a rhythm admits. The fewer equal bi-partitions a rhythm admits, the more rhythmic oddity it possesses. As in [1], this property makes sense only for time spans of even length. Figure 3 shows the three necklace patterns that generate the ten West and South African bell timelines, with all the bi-partitions (in dotted lines) contained in each. The necklaces are labelled with the name of the most representative of its rhythms. We see that the rhythms belonging to the Sorsonet necklace contain three equal bi-partitions, the rhythms belonging to the Tonada necklace contain two equal 
bi-partitions and the rhythms belonging to the Bembé necklace contain one such bi-partition.

The Sorsonet necklace is the least preferred of the three, yielding only two

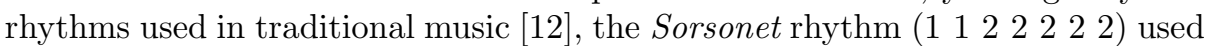
in West Africa and the Persian kitaab al-adwaar rhythm given by (2 222221112$)$. The Tonada necklace is encountered more frequently, yielding two West African

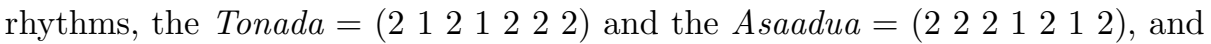

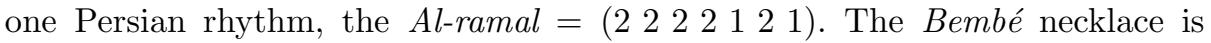
clearly preferred over the other two necklaces. All seven rhythms obtained by starting the cycle at every one of its seven onsets are heavily used [8], [9]. The six listed in the preceeding section are used predominantly in West African, South African, and Afro-American traditional music [7], [12]. The seventh rhythm, given by (1 $\left.2 \begin{array}{lllll}1 & 2 & 2 & 2 & 2\end{array}\right)$, is a Bondo rhythm used in Central Africa [1].

It is evident then that among this family of rhythms there is a marked preference for those that admit as few as possible equal bi-partitions, and thus a high degree of rhythmic oddity. Nevertheless, although this measure performs better than the rhythmic oddity property, it still has limitations. Among the seven rhythms determined by the Bembé necklace some are more popular than others. In fact, one of these, the Bembé $=\left(\begin{array}{llllll}2 & 2 & 1 & 2 & 2 & 2\end{array}\right.$ 1) is by far the most preferred of the seven, and is considered to be the African signature bell-pattern. The master drummer Desmond K. Tai has called it the Standard Pattern [5]. Afro-Cuban music has escorted it across the planet, and it is used frequently on the ride cymbal in jazz. Since all seven rhythms belonging to this necklace have exactly one equal bi-partition, even the multi-valued rhythmic-oddity measure does not discriminate among them, and thus does not favor the Bembe rhythm over the other six.

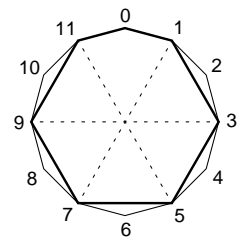

Sorsonet

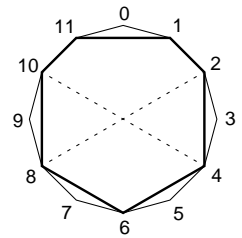

Tonada

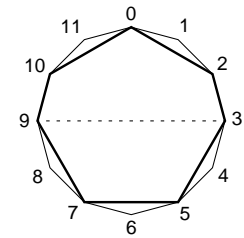

Bembé

Fig. 3. The three necklaces comprising the ten $12 / 8$ time bell patterns. 


\section{The Off-Beatness Measure}

Consider first the rhythms defined in a 12-unit time span. A twelve-unit interval may be evenly divided (with no remainders) by four numbers greater than one and less than twelve. These are the numbers six, four, three and two. Dividing the twelve unit circle by these numbers yields a bi-angle, triangle, square, and hexagon, respectively, as depicted in Figure 4. African music usually incorporates a drum or other percussion instrument on which at least one or portions of these patterns are played. Sometimes the music is accompanied by hand-clapping rhythms that use some or all of these patterns. For example, the Neporo funeral piece of Northwestern Ghana uses the triangle, square, and hexagon clapping patterns [13] shown in Figure 4. In any case the rhythm has a steady fundamental beat which we may associate with position "zero" in the cycle. In polyrhythmic music these four sub-patterns form the possible underlying pulses (sub-beats). Two of the patterns (bi-angle and square) are binary pulses and two (triangle and hexagon) ternary pulses. Therefore notes played in other positions are offbeat in a strong polyrhythmic sense. There are four positions not used by these four pulse patterns. They are positions $1,5,7$, and 11 . Onsets at these positions will be called off-beat onsets. A rhythm that contains at least one off-beat onset will be said to contain the off-beatness property. A measure of the off-beatness of a rhythm is therefore the number of off-beat onsets that it contains.

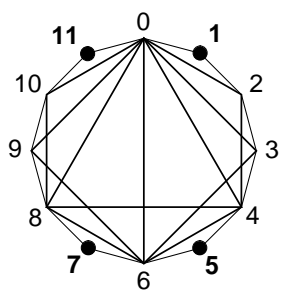

Fig. 4. The four positions not obtainable when dividing 12 by $6,4,3$ or 2 .

These off-beat positions $(1,5,7$, and 11) also have a group-theoretic interpretation. The twelve positions of the possible notes in the cycle form the cyclic group of order 12 denoted by $C_{12}$. The four off-beat position values correspond to the sizes of the intervals that have the property that if one traverses the cycle starting at, say "zero" in a clockwise direction in jumps equal to the size of one of these intervals, then one eventually returns to the starting point after having visited all twelve positions. Conversely, if the lengths of the jumps are taken from the complementary set $(2,3,4,6,8,9,10)$ then the start point will be reached without having visited all twelve positions in the cycle. For this reason the elements $\left(1,5,7\right.$, and 11) are called the generators of the group $C_{12}$. 
Returning to the ten West-African bell patterns in 12/8 time discussed in the preceeding, recall that the Bembé rhythm is the most frequently used of these patterns. Among these ten rhythms, the highest value of off-beatness is three and only the Bembé realizes this value.

Since every cyclic group $C_{n}$ has a set of generators, the off-beatness measure described in the preceeding generalizes to rhythms defined on $n$-unit time spans for other values of $n$. Although the measure works best for even values of $n$, it also has some applicability for odd $n$. On the other hand, if $n$ is some prime number $p$ then every number from 1 to $p-1$ is relatively prime to $p$. In such a case the measure is useless since all the onset positions from 1 to $p-1$ would be off-beatness notes under the present definition of off-beatness. For rhythms that have a 24-unit time span the eight off-beatness onsets are determined by the generators of $C_{24}$, namely, $(1,5,7,11,13,17,19,23)$.

The ten necklaces of nine elements of two colors with density six, of which the Mokongo rhythm of the Aka Pygmies is a member, are listed in Figure 5 along with the number of equal bi-partitions admitted by each. Also included (in the last column) are the off-beatness values of the rhythms in their canonical rotational positions. Of the four rhythms that have the rhythmic oddity property (zero bi-partitions) there are three Lyndon words of interest (shown shaded). Rhythms 7 and 9 have off-beatness values of two, whereas rhythm 8, the Mokongo has a value of three. Hence the off-beatness measure offers an explanation for selecting the Mokongo rhythm over the other two rhythms, both of which have the rhythmic oddity property.

\begin{tabular}{|c|c|c|c|}
\hline No. & Necklace & Bi-Partitions & Off-Beatness \\
\hline 1 & $\begin{array}{lll}333333 & 2222\end{array}$ & 3 & 0 \\
\hline 2 & $\begin{array}{llll}\mathbf{3} 3333 & 2332 & 2\end{array}$ & 2 & 1 \\
\hline 3 & 333332 2232 & 2 & 2 \\
\hline 4 & 333323322 & 1 & 1 \\
\hline 5 & 333323232 & 1 & 2 \\
\hline 6 & 3333322332 & 1 & 1 \\
\hline 7 & 333233322 & 0 & 2 \\
\hline 8 & 333233232 & 0 & 3 \\
\hline 9 & 4333232332 & 0 & 2 \\
\hline 10 & $\begin{array}{lll}333233 & 2332\end{array}$ & 0 & 2 \\
\hline
\end{tabular}

Fig. 5. The ten necklaces of nine elements of two colors with density six.

The number of bi-partitions a rhythm possesses is invariant to cyclic rotations of the rhythm. In other words this property has the same value for a necklace as it does for all the rhythms it generates. By contrast, the off-beatness measure clearly does not have this property. However, there are several ways to obtain an off-beatness measure that is invariant under rotations. One natural method is 
to simply add the off-beatness values over all its onsets used as starting points. This yields an off-beatness value averaged over all starting points. If this is done for the Lyndon words with zero bi-partitions in Figure 5, the Mokongo necklace is still the winner with a score of 27 . Necklace No. 9 comes second with a score of 26 and Necklace No. 7 is third with a score of 24 .

Another (worst-case) rotation-invariant measure of off-beatness, counts the number of onsets that yield the minimum off-beatness value when these onsets are used as starting points in the rhythm. The smaller such a number is, the greater is the guarantee that any starting point of the necklace will yield a rhythm with high off-beatness value. If this number is calculated for necklaces 7, 8, and 9 in Figure 5, the Mokongo necklace is again the winner. The Mokongo necklace (no. 8 in the figure) has one starting onset that yields the minimum off-beatness value of two. Necklace No. 9 comes second with two starting onsets, and necklace No. 7 is third with four such onsets.

\section{Off-Beatness as a Measure of Syncopation}

The off-beatness measure may also serve as a precisely defined mathematical measure of syncopation. According to Michael Keith [6], "although syncopation in music is relatively easy to perceive, it is more than a little difficult to define precisely." Indeed, most definitions have in common that syncopation involves accenting an onset that is not normally accented, which begs for a definition of normal. Keith [6] proposed a mathematical measure of syncopation based on three types of events (notes) he calls hesitation, anticipation, and syncopation, where syncopation is the combination of hesitation and anticipation. To these events he gives the weights 1,2 , and 3 , respectively. An anticipation occurs if the start of the note is "off the beat", whereas a hesitation occurs if the end of the note is "off the beat". What remains to be defined precisely is the notion of "off the beat". Unfortunately Keith defines "off the beat" only for meters with time spans equal to a power of 2 and a partition consisting of all 2's. His definition is as follows:

Let $\delta$ be the duration of the event (note) as a multiple of $1 / 2^{d}$ beats, and let $S$ be the start time of the event (with time positions numbered starting with 0 ), expressed in the same units. Furthermore, let $D=\delta$ rounded down to the nearest power of 2 . Then the start of the event is defined to be "off the beat" if $S$ is not a multiple of $D$, and the end of the event is defined to be "off the beat" if $(S+\delta)$ is not a multiple of $D$. The syncopation value for the $i-t h$ event (note) in the rhythmic pattern, denoted by $s_{i}$ is defined as: $s_{i}=\left(2\right.$ if $s t a r t_{i}$ is off the beat $)+\left(1\right.$ if $e n d_{i}$ is off the beat) Finally, the measure of syncopation of a rhythmic pattern is the sum of the syncopation values $s_{i}$ summed over all $i$.

It is interesting to compare the syncopation measure of Keith with the offbeatness measure proposed here. Since Keith's definition holds only for time spans of units equal to powers of 2 , we cannot compare it to the off-beatness measure using the rhythms of the Aka Pygmies discussed in the preceeding, since they have time spans of 12 and 24 units. However, we may perform the 
comparison using the $4 / 4$ time clave patterns studied in [11] which have time spans of 16 units. The six fundamental timelines are:

$$
\begin{aligned}
& {[\mathrm{x} \ldots \mathrm{x} . \mathrm{x} \ldots \mathrm{x} \cdot \mathrm{x} \ldots]-\text { Shiko }} \\
& {[\mathrm{x} \ldots \mathrm{x} \ldots \mathrm{x} \ldots \mathrm{x} \cdot \mathrm{x} \ldots]-\text { Son }} \\
& {[\mathrm{x} \ldots \mathrm{x} \ldots \mathrm{x} \ldots \mathrm{x} \cdot \mathrm{x} \ldots]-\text { Rumba }} \\
& {[\mathrm{x} \ldots \mathrm{x} \ldots \mathrm{x} \ldots \mathrm{x} \mathrm{x} \ldots]-\text { Soukous }} \\
& {[\mathrm{x} \ldots \mathrm{x} \ldots \mathrm{x} \ldots \mathrm{x} \ldots \mathrm{x} .]-\text { Bossa-Nova }} \\
& {[\mathrm{x} \ldots \mathrm{x} \ldots \mathrm{x} \ldots \mathrm{x} \ldots \mathrm{x} .]-\text { Gahu }}
\end{aligned}
$$

It is also interesting to compare these Afro-American timelines with one of the most popular ostinatos used in classical music given by:

$$
[\mathrm{x} . \mathrm{x} \cdot \mathrm{x} \ldots \mathrm{x} \ldots \mathrm{x} \ldots]-\text { classical ostinato }
$$

\begin{tabular}{|l|c|c|}
\hline & Syncopation & Off-beatness \\
\hline Classical & 0 & 0 \\
\hline Shiko & 1 & 0 \\
\hline Son & 2 & 1 \\
\hline Rumba & 2 & 2 \\
\hline Soukous & 3 & 2 \\
\hline Bossa-Nova & 3 & 2 \\
\hline Gahu & 3 & 1 \\
\hline
\end{tabular}

Fig. 6. A comparison of the syncopation and off-beatness measures.

The values of Syncopation and off-beatness for these seven rhythmic patterns are shown in Figure 6. The syncopation measure makes global sense on these seven rhythms but is rather coarse and yields several questionable judgements. In contrast, the off-beatness measure shows remarkable agreement with human perception of syncopation. There is no doubt that the classical ostinato and Shiko patterns are less syncopated than the Son, Rumba, and Soukous patterns. There is also no doubt that the Bossa-Nova is more syncopated than all of these. Both measures support these conclusions. However, the Rumba is clearly more syncopated than the Son, something the off-beatness measure bears out but the syncopation measure does not. It is difficult to decide which of Soukous or Rumba is the more syncopated of the two. The off-beatness measure reflects this difficulty whereas the syncopation measure judges the Soukous as being more syncopated. The Bossa-Nova feels more syncopated than the Gahu, something the off-beatness measure also bears out but the syncopation measure does not. There appears to be only one point in favor of the syncopation measure. The Shiko is more syncopated than the classical ostinato. Here the syncopation measure agrees more with human perception than does the off-beatness measure. 


\section{Concluding Remarks}

The analysis of Sub-Saharan African rhythm timelines suggests that the offbeatness measure is a good mathematical predictor of the frequency (and thus preference) with which they are used in traditional music. It should be a useful feature for music information retrieval systems, as well as other applications.

The data (4/4 time clave patterns) analysed in the preceeding section suggest that the off-beatness measure also provides more agreement with human perception of syncopation than does the syncopation measure of Keith [6]. Therefore it should be a useful feature for recognizing syncopated music. It would be interesting to generalize Keith's measure to hold for general rhythmic patterns and to compare such a generalization with the off-beatness measure proposed here. Measuring off-beatness for rhythms with time spans consisting of $n$ units, where $n$ is a prime number such as $7,11,13$, etc., is a challenging open problem.

\section{References}

1. Simha Arom. African Polyphony and Polyrhythm. Cambridge University Press, Cambridge, England, 1991.

2. Marc Chemillier. Ethnomusicology, ethnomathematics. The logic underlying orally transmitted artistic practices. In G. Assayag, H. G. Feichtinger, and J. F. Rodrigues, editors, Mathematics and Music, pages 161-183. Springer-Verlag, 2002.

3. Marc Chemillier and Charlotte Truchet. Computation of words satisfying the "rhythmic oddity property" (after Simha Arom's works). Information Processing Letters, 86:255-261, 2003.

4. José Manuel Gamboa. Cante por Cante: Discolibro Didactico de Flamenco. New Atlantis Music, Alia Discos, Madrid, 2002.

5. A. M. Jones. Studies in African Music. Oxford University Press, Amen House, London, 1959.

6. Michael Keith. From Polychords to Pólya: Adventures in Musical Combinatorics. Vinculum Press, Princeton, 1991.

7. Jeff Pressing. Cognitive isomorphisms between pitch and rhythm in world musics: West Africa, the Balkans and Western tonality. Studies in Music, 17:38-61, 1983.

8. Jay Rahn. Asymmetrical ostinatos in sub-saharan music: time, pitch, and cycles reconsidered. In Theory Only, 9(7):23-37, 1987.

9. Jay Rahn. Turning the analysis around: African-derived rhythms and Europederived music theory. Black Music Research Journal, 16(1):71-89, 1996.

10. Frank Ruskey and Joe Sawada. An efficient algorithm for generating necklaces with fixed density. SIAM Journal of Computing, 29(2):671-684, 1999.

11. Godfried T. Toussaint. A mathematical analysis of African, Brazilian, and Cuban clave rhythms. In Proceedings of BRIDGES: Mathematical Connections in Art, Music and Science, pages 157-168, Towson University, Towson, MD, July 27-29 2002.

12. Godfried T. Toussaint. Classification and phylogenetic analysis of African ternary rhythm timelines. In Proceedings of BRIDGES: Mathematical Connections in Art, Music and Science, pages 25-36, Granada, Spain, July 23-27 2003.

13. Trevor Wiggins. Techniques of variation and concepts of musical understanding in Northern Ghana. British Journal of Ethnomusicology, 7:117-142, 1998. 